\title{
About the Benjamin-Bona-Mahony Equation in Domains with Moving Boundary
}

C.S.Q. CALDAS, J. LIMACO, R.K. BARRETO, ${ }^{3}$ Departamento de Matemática Aplicada, Instituto de Matemática, UFF, Rua Mário Santos Braga s/n, 24020-140 Niterói, RJ, Brasil

P. GAMBOA, Instituto de Matemática, UFRJ, Cx.P. 68530, Av. Brigadeiro Trompowski s/n, 21945-970 Rio de Janeiro, RJ, Brasil.

\begin{abstract}
In this article, we prove the existence of solutions for an hyperbolic equation known as the Benjamin-Bona-Mahony equation. Our study involves increasing, decreasing, and mixed non-cylindrical domains and for this analysis, our main tools are the change of variable technique, the Galerkin and penalization method.
\end{abstract}

Key Words. Benjamin-Bona-Mahony equation, noncylindrical domains, change of variable technique.

\section{Introduction}

Let $\alpha, \beta \in C^{2}([0, T] ; \mathbf{R})$, such that $\alpha(t)<\beta(t)$, for all $0 \leq t \leq T$. We represent by $\hat{Q}$ the noncylindrical domain of $\mathbf{R}^{\mathbf{2}}$, given by $\hat{Q}=\left\{(x, t) \in \mathbf{R}^{\mathbf{2}} ; \alpha(t)<x<\beta(t)\right.$, $\forall 0<t<T\}$, with lateral boundary $\sum^{\wedge}$ defined by $\sum^{\wedge}=\bigcup_{0<t<T}\{\alpha(t), \beta(t)\} \times\{t\}$ and supposed $\widehat{Q} \subset Q=\Omega \times] 0, T[$, where $\Omega$ denotes $] 0,1[$.

In the present work we investigate the existence, of solutions for the following problem

$$
\mid \begin{array}{ll}
u_{t}+(\sigma(u))_{x}-u_{x x t}=0 & \text { in } \widehat{Q} \\
u(x, t)=0 & \text { for }(x, t) \in \widehat{\sum} \\
u(x, 0)=u^{0}(x) & \text { in } \alpha(0)<x<\beta(0),
\end{array}
$$

where $\sigma: \mathbf{R} \rightarrow \mathbf{R}$ is a $\mathbf{C}^{1}$-function. such that

\footnotetext{
1 gmacruz@vm.uff.br

${ }_{2}^{2}$ jlimaco@vm.uff.br

3 rikaba@vm.uff.br

${ }^{4}$ pgamboa@im.ufrj.br
} 
(H1) There exist constants $\sigma_{0}, \rho>0$ such that $\left|\sigma^{\prime}(\lambda)\right| \leq \sigma_{0}\left(1+|\lambda|^{\rho-1}\right)$.

(H2) There exists constant $\sigma_{1}>0$ such that $|\sigma(\lambda)| \leq \sigma_{1}\left(|\lambda|+|\lambda|^{\rho}\right)$.

Benjamin, Bona \& Mahony in [3] introduced the equation

$$
u_{t}(x, t)+u_{x}(x, t)+u(x, t) u_{x}(x, t)-u_{x x t}(x, t)=0 \text { in } \mathbf{R} \times[0, T],
$$

which is an alternative smoothness model for the $\mathrm{KdV}$, known as Benjamin-BonaMahony equation or BBM equation. In [3], the existence and uniqueness of solutions for the Cauchy problem associated with (1.2) were investigated. Subsequently, Bona and Briant [4] investigated the BBM equations with Dirichlet boundary conditions, considering the spatial variable $x \in[0, \infty]$. Medeiros \& Milla Miranda [12] studied the existence, uniqueness and regularity of solutions for the BBM equation with general nonlinear term when $x \in] 0,1$ [. Later was the time for Bona \& Dougalis [5] prove the existence and uniqueness of solutions for the BBM equation considering non-homogeneous boundary conditions. The case $n$ dimensional of the equation (1.2) was studied by Goldstein [8] and again in the work of Avrin and Goldstein together [1]. Similar equations to (1.2) in noncylindrical domains were studied by Cousin and Larkin for the Kuramoto Sivashinski equation [7] and recently Barreto et al. [2] for the Rosenau and BBM equations. To study the Rosenau equation in cylindrical domains see Park [13] and Rosenau [14]. In this article, we study the existence of solutions for the problem (1.1) for increasing, decreasing and mixed noncylindrical domains. The importance of our work can be noticed, as the change of variable technique makes the computational study of the problem in noncylindrical domains easier. Some works that we know in this direction are Liu and Rincon [10] that use the paper Medeiros and Limaco [11] and Santos et al. [15], that use Caldas et al. [6].

This paper is organized as follows. The next section is devoted to the existence and uniqueness of solution for (1.1), satisfying the hypothesis:

$(H 3) \quad \alpha^{\prime}(t) \geq 0$, and $\beta^{\prime}(t) \leq 0$ for $t \in[0, T]$.

Note that this hypothesis implies that $\widehat{Q}$ decreases in a sense such that if $t_{2}>t_{1}$, then the projection of $\left[\alpha\left(t_{2}\right), \beta\left(t_{2}\right)\right]$ in the subspace $t=0$ is contained in the projection of $\left[\alpha\left(t_{1}\right), \beta\left(t_{1}\right)\right]$ in the same subspace.

In the third section of this article, we study the existence of solutions for (1.1) satisfying the hypothesis:

$(H 4) \alpha^{\prime}(t) \leq 0$ and $\beta^{\prime}(t) \geq 0$ for $t \in[0, T]$.

Analogously, hypothesis $(H 4)$ implies that $\widehat{Q}$ increases.

In the last section of this article we study (1.1), satisfying the hypothesis: 


$$
\begin{gathered}
\widehat{Q}=\widehat{Q}_{1} \cup \widehat{Q}_{2} \cup\left\{\left(x, T_{1}\right) \in \mathbf{R}^{2} ; \quad \alpha\left(T_{1}\right)<x<\beta\left(T_{1}\right)\right\} \\
\quad \text { where } \\
\widehat{Q}_{1}=\left\{(x, t) \in \mathbf{R}^{2} ; \alpha(t)<x<\beta(t), 0<t<T_{1}\right\} \\
\quad \text { is decreasing satisfying }(H 1) \text { and } \\
\widehat{Q}_{2}=\left\{(x, t) \in \mathbf{R}^{2} ; \alpha(t)<x<\beta(t), T_{1}<t<T\right\}
\end{gathered}
$$
is increasing satisfying $(H 2)$.

In the following, by $\Omega_{t}$ and $\Omega_{0}$ denote the intervals $] \alpha(t), \beta(t)[$ and $] \alpha(0), \beta(0)[$ respectively; we denote, by $(.,$.$) , \|$.$\| respectively the scalar product and norm in$

$L^{2}(\Omega)$. We also denote, $\frac{\partial w_{m}}{\partial x}, \frac{\partial^{2} w_{m}}{\partial x^{2}}, \frac{\partial^{2} w_{m}}{\partial t \partial x}$, by $w_{m, x}, w_{m, x x}, w_{m, x t}$ respectively, etc.

\section{Solution on Decreasing Domains}

In this section we study the existence and uniqueness for (1.1) satisfying $(H 1)$ and $(H 3)$.

Let be $\gamma(t)=\beta(t)-\alpha(t)>0$, for all $t \geq 0$. Then $0<\frac{x-\alpha(t)}{\gamma(t)}<1$, for all $t \in[0, T]$. With the change of variable $u(x, t)=v(y, t)$ with $y=\frac{x-\alpha(t)}{\gamma(t)}$, for all $t \in[0, T]$, problem $(1.1)$ is transformed into

$$
\mid \begin{array}{lll}
v_{t}+\frac{1}{\gamma}(\sigma(v))_{y}-\frac{1}{\gamma^{2}} v_{y y t}-\frac{\left(\alpha^{\prime}+\gamma^{\prime} y\right)}{\gamma} v_{y}+\frac{2 \gamma^{\prime}}{\gamma^{3}} v_{y y} & \\
+\frac{\left(\alpha^{\prime}+\gamma^{\prime} y\right)}{\gamma^{3}} v_{y y y}=0 & \text { in } & \Omega \times] 0, T[ \\
v(0, t)=v(1, t)=0 & \text { in } \quad] 0, T[ \\
v(y, 0)=v^{0}(y) & \text { in } \quad \Omega .
\end{array}
$$

In these conditions, we can establish the following existence result:

Theorem 2.1. Given $u^{0} \in H_{0}^{1}\left(\Omega_{0}\right) \cap H^{2}\left(\Omega_{0}\right)$, there exists a unique function $u: \widehat{Q} \rightarrow \mathbf{R}$, satisfying $u \in L^{\infty}\left(0, T ; H_{0}^{1}\left(\Omega_{t}\right)\right), u_{t} \in L^{\infty}\left(0, T ; H_{0}^{1}\left(\Omega_{t}\right)\right)$ and

$$
\int_{\widehat{Q}} u_{t} \phi d x d t+\int_{\widehat{Q}}(\sigma(u))_{x} \phi d x d t+\int_{\widehat{Q}} u_{x t} \phi_{x} d x d t=0
$$

for all $\phi \in L^{2}\left(0, T ; H_{0}^{1}\left(\Omega_{t}\right)\right) \quad u(x, 0)=u^{0}(x)$, for all $x \in \Omega_{0}$.

In order to prove Theorem 2.1, we need the following lemma:

Lemma 2.1. Given $v^{0} \in H_{0}^{1}(\Omega) \cap H^{2}(\Omega)$, there exists a unique function $v: \Omega \times] 0, T\left[\longrightarrow \mathbf{R}\right.$, satisfying $v \in L^{\infty}\left(0, T ; H_{0}^{1}(\Omega) \cap H^{2}(\Omega)\right), v_{t} \in L^{\infty}\left(0, T ; H_{0}^{1}(\Omega)\right)$, 
and

$$
\begin{gathered}
\int_{\Omega \times] 0, T[}\left[v_{t} \psi+\frac{1}{\gamma}(\sigma(v))_{y} \psi+\frac{1}{\gamma^{2}} v_{y t} \psi_{y}-\frac{\left(\alpha^{\prime}+\gamma^{\prime} y\right)}{\gamma} v_{y} \psi+\frac{2 \gamma^{\prime}}{\gamma^{3}} v_{y y} \psi\right. \\
\left.-\left(\frac{\left(\alpha^{\prime}+\gamma^{\prime} y\right) \psi}{\gamma^{3}}\right)_{y} v_{y y}\right] d y d t=0, \quad \text { for all } \psi \in L^{2}\left(0, T ; H_{0}^{1}(\Omega)\right) \\
v(y, 0)=v^{0}(y), \quad \text { for all } \quad y \in \Omega .
\end{gathered}
$$

Proof. Let $\left(w_{i}\right)_{i \in \mathbf{N}}$ be the special basis of $H_{0}^{1}(\Omega)$, such that

$$
\begin{aligned}
w_{i, y y} & =\lambda_{i} w_{i} \quad \text { in } \Omega \\
w_{i}(0) & =w_{i}(1)=0 \quad i \in \mathbf{N} .
\end{aligned}
$$

We denote by $V_{m}$ the subspace generated by $w_{1}, \ldots, w_{m}$. We start by constructing the Galerkin approximation of the solution $v_{m} \in V_{m}$, which is given by the solution of the approximate equation:

$$
\mid \begin{aligned}
& \left(v_{m, t}(t), w\right)+\frac{1}{\gamma}\left(\left(\sigma\left(v_{m}(t)\right)_{y}, w\right)-\frac{1}{\gamma^{2}}\left(v_{m, y y t}(t), w\right)\right. \\
& -\left(\frac{\left(\alpha^{\prime}+\gamma^{\prime} y\right)}{\gamma} v_{m, y}(t), w\right) \\
& +\frac{2 \gamma^{\prime}}{\gamma^{3}}\left(v_{m, y y}(t), w\right)+\left(\frac{\left(\alpha^{\prime}+\gamma^{\prime} y\right)}{\gamma^{3}} v_{m, y y y}(t), w\right)=0 \quad \text { for all } w \in V_{m} \\
& v_{m}(0)=v_{m}^{0} \longrightarrow v^{0} \quad \text { in } H^{2}(0,1) .
\end{aligned}
$$

Note that the solution $v_{m}$ defined on $\left[0, t_{m}\right.$ [ can be extended to the interval $[0, T]$, by the next first a priori estimate.

First Estimate. Taking $w=2 v_{m}(t)$ in (2.4), we have

$$
\begin{aligned}
& \frac{d}{d t}\left(\left\|v_{m}(t)\right\|^{2}+\frac{1}{\gamma^{2}}\left\|v_{m, y}(t)\right\|^{2}\right)+\frac{\gamma^{\prime}}{\gamma}\left\|v_{m}(t)\right\|^{2}+\frac{\gamma^{\prime}}{\gamma^{3}}\left\|v_{m, y}(t)\right\|^{2} \\
& +\frac{\alpha^{\prime}}{\gamma^{3}} v_{m, y}^{2}(0)-\frac{\beta^{\prime}}{\gamma^{3}} v_{m, y}^{2}(1)+\frac{1}{\gamma}\left(\sigma\left(v_{m}(t)\right)_{y}, 2 v_{m}(t)\right)=0 .
\end{aligned}
$$

Since

$$
\left(\sigma\left(v_{m}(t)\right)_{y}, 2 v_{m}(t)\right)=-2 \int_{0}^{1} \frac{d}{d y}\left[\bar{\sigma}\left(v_{m}(t)\right)\right] d y=0,
$$

where $\bar{\sigma}(s)=\int_{0}^{s} \sigma(\lambda) d \lambda$, then from (2.5), (2.6) and (H1), there exist $C_{1}>0$, such that

$$
\begin{aligned}
\frac{d}{d t}\left(\left\|v_{m}(t)\right\|^{2}\right. & \left.+\frac{1}{\gamma^{2}}\left\|v_{m, y}(t)\right\|^{2}\right)+\frac{\alpha^{\prime}}{\gamma^{3}} v_{m, y}^{2}(0)-\frac{\beta^{\prime}}{\gamma^{3}} v_{m, y}^{2}(1) \\
& \leq C_{1}\left(\left\|v_{m}(t)\right\|^{2}+\left\|v_{m, y}(t)\right\|^{2}\right) .
\end{aligned}
$$


Integrating (2.7) over $[0, t]$ with $0<t<t_{m}$ and using hypothesis $(H 3)$, we obtain

$$
\begin{aligned}
\left\|v_{m}(t)\right\|^{2} & +\frac{1}{\gamma^{2}}\left\|v_{m, y}(t)\right\|^{2} \leq\left\|v^{0}\right\|^{2}+\frac{1}{\gamma^{2}(0)}\left\|v_{y}^{0}\right\|^{2} \\
& +C_{1} \int_{0}^{t}\left(\left\|v_{m}(s)\right\|^{2}+\left\|v_{m, y}(s)\right\|^{2}\right) d s
\end{aligned}
$$

From (2.8) and Gronwall inequality, we obtain

$$
\left\|v_{m}(t)\right\|^{2}+\frac{1}{\gamma^{2}}\left\|v_{m, y}(t)\right\|^{2} \leq C_{2}
$$

where $C_{2}, C_{3}, \ldots$ denote positive constants.

Second Estimate. Taking $w=-2 v_{m, y y}(t)$ in $(2.4)$, we have

$$
\begin{aligned}
& \frac{d}{d t}\left[\left\|v_{m, y}(t)\right\|^{2}+\frac{1}{\gamma^{2}}\left\|v_{m, y y}(t)\right\|^{2}\right]-\frac{\gamma^{\prime}}{\gamma^{3}}\left\|v_{m, y y}(t)\right\|^{2}+\frac{\alpha^{\prime}}{\gamma^{3}} v_{m, y y}^{2}(0) \\
& -\frac{\beta^{\prime}}{\gamma^{3}} v_{m, y y}^{2}(1)=\frac{2}{\gamma}\left(\left(\sigma\left(v_{m}(t)\right)_{y}, v_{m, y y}(t)\right)-\left(\frac{\left(\alpha_{1}+\gamma^{\prime} y\right)}{\gamma} v_{m, y}(t), 2 v_{m, y y}(t)\right) .\right.
\end{aligned}
$$

From (2.10) and using Young's inequality and Poincare's inequality, we get

$$
\begin{aligned}
& \frac{d}{d t}\left[\left\|v_{m, y}(t)\right\|^{2}+\frac{1}{\gamma^{2}}\left\|v_{m, y y}(t)\right\|^{2}\right]+\frac{\alpha^{\prime}}{\gamma^{3}} v_{m, y y}^{2}(0) \\
& -\frac{\beta^{\prime}}{\gamma^{3}} v_{m, y y}^{2}(1) \leq C_{3}\left(\left\|v_{m, y}(t)\right\|^{2}+\frac{1}{\gamma 2}\left\|v_{m, y y}(t)\right\|^{2}\right) .
\end{aligned}
$$

Integrating (2.11) over $[0, t]$, using hypothesis $(H 1)$ and applying Gronwall inequality, we have:

$$
\left\|v_{m, y}(t)\right\|^{2}+\frac{1}{\gamma^{2}}\left\|v_{m, y y}(t)\right\|^{2} \leq C_{4} .
$$

Third Estimate. Taking $w=v_{m, t}(t)$ in (2.4), we have:

$$
\begin{aligned}
& \left\|v_{m, t}(t)\right\|^{2}+\frac{1}{\gamma^{2}}\left\|v_{m, y t}(t)\right\|^{2}=-\frac{1}{\gamma}\left(\sigma^{\prime}\left(v_{m}(t)\right) v_{m, y}(t), v_{m, t}(t)\right) \\
& +\left(\frac{\left(\alpha^{\prime}+\gamma^{\prime} y\right)}{\gamma} v_{m, y}(t), v_{m, t}(t)\right)-\frac{2 \gamma^{\prime}}{\gamma^{3}}\left(v_{m, y y}(t), v_{m, t}(t)\right) \\
& +\frac{\gamma^{\prime}}{\gamma^{3}}\left(v_{m, y y}(t), v_{m, t}(t)\right)+\left(\frac{\left(\alpha^{\prime}+\gamma^{\prime} y\right)}{\gamma^{3}} v_{m, y y}(t), v_{m, y t}(t)\right)
\end{aligned}
$$

From (2.10), (2.12) and (2.13), we have

$$
\left\|v_{m, t}(t)\right\|^{2}+\frac{1}{\gamma^{2}}\left\|v_{m, y t}(t)\right\|^{2} \leq C_{5}
$$

Estimates (2.9), (2.13) and (2.14), allow us to pass to the limit in (2.4) and we obtain a weak solution $v$ in the sense of Lemma 2.1 .

The uniqueness of solution and the verification of initial data are shown by the standard arguments.

The Proof of Theorem 2.1. follows immediately from Lemma 2.2 and the Change of Variable Theorem. Therefore, we omit it. 


\section{Solutions on Increasing Domains}

In this section we study the existence of solution for the systems (1.1) satisfying the hypotheses $(H 2)$ and $(H 4)$. We use the Penalization Method given by Lions [9]. Let $Q=] a, b[\times] 0, T[$ be a cylinder such that $Q \supset \widehat{Q}$. We define the function $M: Q \longrightarrow \mathbf{R}$, by $M(x, t)=\left\{\begin{array}{l}\left.1 \text { in } Q \backslash \widehat{Q} \cup\left(\Omega_{0} \times\{0\}\right)\right\} \\ 0 \text { in } \widehat{Q} \cup\left(\Omega_{0} \times\{0\}\right) .\end{array}\right.$

To show the existence of result we will use the following Lemma:

Lemma 3.1. Under the hypothesis (H4), if $u, u_{t} \in L^{2}\left(0, T ; L^{2}(a, b)\right)$, then

$$
\int_{0}^{t}\left(M u(s), u_{t}(s)\right) d s \geq \frac{1}{2}\|M(t) u(t)\|_{L^{2}(a, b)}^{2}-\frac{1}{2}\|M(0) u(0)\|_{L^{2}(a, b)}^{2} .
$$

Proof. We have

$$
\begin{aligned}
\int_{0}^{t}\left(M u(s), u_{t}(s)\right) d s & =\frac{1}{2} \int_{0}^{t} \int_{a}^{b} M\left(u^{2}(s)\right)_{t} d \xi d s \\
& =\frac{1}{2} \int_{[0, t] \times[a, b]} M\left(u^{2}(s)\right)_{t} d \xi d s
\end{aligned}
$$

From Fubini's Theorem, recalling the definition of $M$, and the hypothesis (H4), it follows that

$$
\begin{aligned}
& \int_{0}^{t}\left(M u(s), u_{t}(s)\right) d s \\
= & \frac{1}{2} \int_{a}^{\alpha(t)} \int_{0}^{t}\left[u^{2}(s)\right]_{t} d s d \xi+\frac{1}{2} \int_{\alpha(t)}^{\alpha(0)} \int_{0}^{\alpha^{-1}(x)}\left[u^{2}(s)\right]_{t} d s d \xi \\
& +\frac{1}{2} \int_{\beta(0)}^{\beta(t)} \int_{0}^{\beta^{-1}(x)}\left[u^{2}(s)\right]_{t} d s d \xi+\frac{1}{2} \int_{\beta(t)}^{b} \int_{0}^{t}\left[u^{2}(s)\right]_{t} d s d \xi \\
= & \frac{1}{2} \int_{a}^{\alpha(t)}\left[u^{2}(t, \xi)-u^{2}(0, \xi)\right] d \xi+\frac{1}{2} \int_{\alpha(t)}^{\alpha(0)}\left[u^{2}\left(\alpha^{-1}(x), 0\right)-u^{2}(0, \xi)\right] d \xi \\
& +\frac{1}{2} \int_{\beta(0)}^{\beta(t)}\left[u^{2}\left(\beta^{-1}(x), 0\right)-u^{2}(0, \xi)\right] d \xi+\frac{1}{2} \int_{\beta(t)}^{b}\left[u^{2}(t, \xi)-u^{2}(0, \xi)\right] d \xi
\end{aligned}
$$




$$
\begin{aligned}
& \geq \frac{1}{2}\left[\int_{a}^{\alpha(t)} u^{2}(t, \xi) d \xi+\int_{\beta(t)}^{b} u^{2}(t, \xi) d \xi-\left(\int_{a}^{\alpha(t)} u^{2}(0, \xi) d \xi\right.\right. \\
& \left.\left.\quad+\int_{\alpha(t)}^{\alpha(0)} u^{2}(0, \xi) d \xi+\int_{\beta(0)}^{\beta(t)} u^{2}(0, \xi) d \xi+\int_{\beta(t)}^{b} u^{2}(0, \xi) d \xi\right)\right] \\
& =\frac{1}{2} \int_{a}^{b} M(t, \xi) u^{2}(t, \xi) d \xi-\frac{1}{2} \int_{a}^{b} M(0, \xi) u^{2}(0, \xi) d \xi \\
& =\frac{1}{2}\|M(t) u(t)\|_{L^{2}(a, b)}^{2}-\frac{1}{2}\|M(0) u(0)\|_{L^{2}(a, b)}^{2}
\end{aligned}
$$

which completes the proof.

The existence of solution for (1.1), satisfying the hypothesis $(H 2)$ and $(H 4)$, is established in the next theorem.

Theorem 3.1. For each $u^{0} \in H_{0}^{1}\left(\Omega_{0}\right)$, there exists a function $u: \widehat{Q} \rightarrow \mathbf{R}$, satisfying $u \in L^{\infty}\left(0, T ; H_{0}^{1}\left(\Omega_{t}\right)\right), u_{t} \in L^{\infty}\left(0, T ; H_{0}^{1}\left(\Omega_{t}\right)\right)$ and

$$
\begin{aligned}
& \int_{\widehat{Q}} u_{t} \phi d x d t-\int_{\widehat{Q}} \sigma(u) \phi_{x} d x d t+\int_{\widehat{Q}} u_{x t} \phi_{x} d x d t=0, \\
& \text { for all } \varphi \in L^{2}\left(0, T ; H_{0}^{1}\left(\Omega_{t}\right)\right) \\
& u(x, 0)=u^{0}(x), \quad \text { for all } x \in \Omega_{0} .
\end{aligned}
$$

Proof. To prove this result we will use the penalization method. For each $\varepsilon>0$ we consider the following problem:

$$
\mid \begin{array}{ll}
u_{\varepsilon, t}+\left(\sigma\left(u_{\varepsilon}\right)\right)_{x}-u_{\varepsilon, x x t}+\frac{1}{\varepsilon} M u_{\varepsilon, t}=0 & \text { in } Q \\
u_{\varepsilon}(a, t)=u_{\varepsilon}(b, t)=0 & \text { for }] 0, T[ \\
u_{\varepsilon}(x, 0)=\widetilde{u}^{0}(x) & \text { in }] a, b[
\end{array}
$$

Let $\left\{w_{i}\right\}_{i \in N}$ be a basis of $H_{0}^{1}(a, b)$, such that $w_{1}=\widetilde{u}_{0}$. We denote by $V_{m}=$ $\left[w_{1}, \ldots, w_{m}\right]$ the subspace of $H_{0}^{1}(a, b)$, generated by $\widetilde{u}_{0}, w_{2}, \ldots, w_{m}$. We seek $u_{\varepsilon m}(t) \in$ $V_{m}$ solution of the following approximate problem:

$$
\mid \begin{array}{ll}
\left.\left(u_{\varepsilon m, t}(t), w\right)+\left(\sigma\left(u_{\varepsilon m}(t)\right)\right)_{x}, w\right)-\left(u_{\varepsilon m, x x t}(t), w\right) & \\
+\frac{1}{\varepsilon}\left(M(t) u_{\varepsilon m, t}(t), w\right)=0 & \text { for all } w \in V_{m} \\
u_{\varepsilon m}(0)=u^{0}(x) \longrightarrow \widetilde{u}^{0} & \text { in } H_{0}^{1}(a, b) .
\end{array}
$$

By the Caratheodory theorem, the system (3.3) has solutions on some interval $\left[0, t_{m}[\right.$. To extend them to any finite interval and to pass to the limit as $m \rightarrow \infty$ and $\varepsilon>0$ fixed, we need a priori estimates. 
First Estimate. Taking $w=2 u_{\varepsilon m}(t)$ in (3.3), we have

$$
\begin{aligned}
\frac{d}{d t}\left[\|\| u_{\varepsilon m}(t)\|\|^{2}\right. & \left.+\left\|u_{\varepsilon m, x}(t)\right\| \|^{2}\right]+\left(\left(\sigma\left(u_{\varepsilon m}(t)\right)\right)_{x}, w\right) \\
& +\frac{2}{\varepsilon}\left(M(t) u_{\varepsilon m, t}(t), w\right)=0,
\end{aligned}
$$

where $\||||| \mid$ denotes the norm in $L^{2}(a, b)$.

Using the Fundamental Theorem of Calculus and integrating by parts we obtain:

$$
\int_{a}^{b}\left[\sigma\left(u_{\varepsilon m}(t)\right)\right]_{x} u_{\varepsilon m}(t) d x=0 .
$$

Integrating (3.4) over $[0, t]$, using (3.5) and Lemma 3.1, we have

$$
\begin{aligned}
\left.\left\|u_{\varepsilon m}(t)\right\|\right|^{2} & +\left.\left\|u_{\varepsilon m, x}(t)\right\|\right|^{2}+\frac{1}{\varepsilon}\left\|M(t) u_{\varepsilon m}(t)\right\| \|^{2} \\
& \leq\left.\left\|\widetilde{u^{0}}\right\|\right|^{2}+\left\|\widetilde{u_{x}^{0}}\right\| \|^{2} \leq C_{6} .
\end{aligned}
$$

Second Estimate. Taking $w=u_{\varepsilon m, t}(t)$ in (3.3) and using (H2) and (3.6), we have:

$$
\begin{aligned}
\left.\left\|u_{\varepsilon m, t}(t)\right\|\right|^{2} & +\left\|\mid u_{\varepsilon m, x t}(t)\right\| \|^{2}+\frac{1}{\varepsilon}\left(M(t) u_{\varepsilon m, t}(t), u_{\varepsilon m, t}(t)\right) \\
& \leq C_{7}+\frac{1}{2}\|\| u_{\varepsilon m, t}(t)\|\|^{2} .
\end{aligned}
$$

From (3.7) we obtain

$$
\frac{1}{2}\left\|\left|u_{\varepsilon m, t}(t)\left\|\left.\right|^{2}+\right\|\right| u_{\varepsilon m, x t}(t)\right\|^{2}+\frac{1}{\varepsilon}\left\|\left|M(t) u_{\varepsilon m, t}(t) \|\right|^{2} \leq C_{8} .\right.
$$

From the estimates (3.6) and (3.8) we pass to limit in (3.3), and we obtain that $u_{\varepsilon}$ is a solution of the penalized problem

$$
\begin{aligned}
& \int_{0}^{T} \int_{a}^{b} u_{\varepsilon, t} \phi d x d t+\int_{0}^{T} \int_{a}^{b}\left(\sigma\left(u_{\varepsilon}\right)\right)_{x} \phi d x d t+\int_{0}^{T} \int_{a}^{b} u_{\varepsilon, x t} \phi_{x} d x d t \\
& +\frac{1}{\varepsilon} \int_{0}^{T} \int_{a}^{b} M u_{\varepsilon, t} \phi d x d t=0 \quad \text { for all } \phi \in L^{2}\left(0, T ; H_{0}^{1}(a, b)\right) .
\end{aligned}
$$

From (3.6), (3.8) and the Banach-Steinhauss Theorem, we pass to the limit as $\varepsilon \rightarrow 0$ in (3.9) and we obtain (3.1).

Regularity. From (3.6), we have

$$
\frac{1}{\varepsilon} \int_{0}^{t}\left(M(s) u_{\varepsilon m, t}(s), u_{\varepsilon m}(s)\right) d s \leq C_{9} .
$$

On the other hand, from Lemma 3.1 we obtain:

$$
\frac{1}{\varepsilon} \int_{0}^{t}\left(M(s) u_{\varepsilon m, t}(s), u_{\varepsilon m}(s)\right) d s \geq \frac{1}{2 \varepsilon}\|\| M(t) u_{\varepsilon m}(t) \|^{2} .
$$


Then $\left.\left\|M(t) u_{\varepsilon m}(t)\right\|\right|^{2} \leq 2 C_{9} \varepsilon$. Thus $\int_{0}^{T} \int_{a}^{b} M(t) u_{\varepsilon m}^{2}(t) d x d t \leq 2 C_{9} \varepsilon T$ or

$$
\int_{0}^{T} \int_{a}^{b}\left|M(t) u_{\varepsilon}(t)\right|^{2} d x d t \leq \liminf \int_{0}^{T} \int_{a}^{b}\left|M(t) u_{\varepsilon}(t)\right|^{2} d x d t \leq 2 C_{9} \varepsilon T .
$$

Then $M u_{\varepsilon} \rightarrow 0$ strong in $L^{2}\left(0, T ; L^{2}(a, b)\right)$, as $\varepsilon \rightarrow 0^{+}$. On the other hand, $M u_{\varepsilon} \rightarrow M u$ in $L^{2}\left(0, T ; L^{2}(a, b)\right)$ and $M u_{\varepsilon m} \rightarrow M u_{\varepsilon}$ in $L^{2}\left(0, T ; L^{2}(a, b)\right)$, as $\varepsilon \rightarrow 0^{+}$.

Therefore, we conclude that: $M u=0$ a.e. in $Q$ or $u=0$ in $Q \backslash \widehat{Q}$.

Since $u \in L^{\infty}\left(0, T ; H_{0}^{1}(a, b)\right)$ and $u_{t} \in L^{\infty}\left(0, T ; H_{0}^{1}(a, b)\right)$, then $u \in C([0, T]$; $\left.H_{0}^{1}(a, b)\right)$. Thus, $u(t) \in H_{0}^{1}(a, b)$ for all $t$ and $u=0$ in $] a, b[\backslash] \alpha(t), \beta(t)[$. From there, we have that $u(t) \in H_{0}^{1}(\alpha(t), \beta(t))$, for all $t$. Thus $u \in L^{\infty}\left(0, T ; H_{0}^{1}\left(\Omega_{t}\right)\right)$.

From the second estimate, we have

$$
\int_{0}^{T} \int_{a}^{b}\left|M(t) u_{\varepsilon m, t}(t)\right|^{2} d x d t \leq 2 c \varepsilon T
$$

From (3.10) and by similar arguments, we obtain that $u_{t} \in L^{\infty}\left(0, T ; H_{0}^{1}\left(\Omega_{t}\right)\right)$, which prove the regularity of the solution.

Remark 3.1. Theorems 2.1 and 3.1 are invariable by translation.

In fact, the particular problem

$$
\mid \begin{array}{ll}
u_{t}+(\sigma(u))_{x}-u_{x x t}=0 & \text { in } \widehat{Q} \subset \Omega \times] T_{0}, T_{1}[ \\
u(x, t)=0 & \text { in } \widehat{\sum} \\
u\left(x, T_{0}\right)=u^{0}(x) & \text { in } \Omega_{T_{0}} .
\end{array}
$$

with the change of variable $u(x, t)=\bar{u}\left(x, t-T_{0}\right)$ can be transformed into a problem of the type (1.1).

\section{Solutions on Mixed Domains}

Here we analyze the case where $\widehat{Q}$ is a mixed domain, satisfying the hypothesis (H5). Let $\widehat{\sum}_{1}$ and $\widehat{\sum}_{2}$ the lateral boundaries of $\widehat{Q}_{1}$ and $\widehat{Q}_{2}$ respectively.

To find a solution of (1.1) in $\widehat{Q}$, we consider the following two cases:

1) Solution on $\widehat{Q}_{1}$. For each $u^{0} \in H_{0}^{1}\left(\Omega_{0}\right) \cap H^{2}\left(\Omega_{0}\right)$, by Theorem 2.1 , there exists $u_{1}$ solution of

$$
\mid \begin{array}{ll}
u_{1, t}+\left(\sigma\left(u_{1}\right)\right)_{x}-u_{1, x x t}=0 & \text { in } \widehat{Q}_{1} \\
u_{1}(x, t)=0 & \text { in } \widehat{\sum}_{1} \\
u_{1}(x, 0)=u^{o}(x) & \text { in } \Omega_{0}
\end{array}
$$


satisfying $u_{1} \in L^{\infty}\left(0, T_{1} ; H_{0}^{1}\left(\Omega_{t}\right)\right), u_{1 t} \in L^{\infty}\left(0, T_{1} ; H_{0}^{1}\left(\Omega_{t}\right)\right)$. Therefore $u_{1}$ is in $C\left(\left[0, T_{1}\right] ; H_{0}^{1}\left(\Omega_{t}\right)\right)$.

2) Solution on $\widehat{Q}_{2}$

For each $\bar{u}^{0}=u_{1}\left(T_{1}\right) \in H_{0}^{1}\left(\Omega_{T_{1}}\right)$, by Theorem 3.1 and Remark 3.1, there exists $u_{2}$ solution of

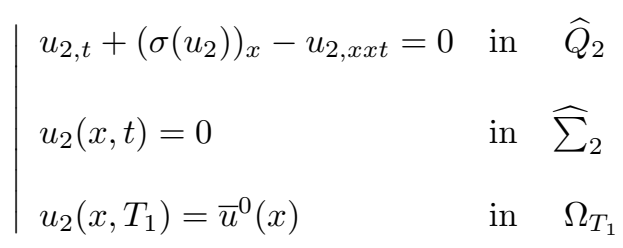

satisfying $u_{2} \in L^{\infty}\left(T_{1}, T ; H_{0}^{1}\left(\Omega_{t}\right)\right), u_{2 t} \in L^{\infty}\left(T_{1}, T ; H_{0}^{1}\left(\Omega_{t}\right)\right), u_{2} \in C\left(\left[T_{1}, T\right] ; H_{0}^{1}\left(\Omega_{t}\right)\right)$. 3) Solution on $\widehat{Q}$

We define $u: \widehat{Q} \longrightarrow \mathbf{R}$ by $u(x, t)= \begin{cases}u_{1}(x, t), & (x, t) \in \widehat{Q}_{1} \\ u_{2}(x, t), & (x, t) \in \widehat{Q}_{2}\end{cases}$ where $u_{1}$ and $u_{2}$ are solutions of (4.1) and (4.2), respectively. Given that $u^{0} \in$ $H_{0}^{1}\left(\Omega_{0}\right)$, by Remark 3.1, we deduce that the function $u$ defined above is solution of:

$$
\mid \begin{array}{ll}
u_{t}+(\sigma(u))_{x}-u_{x x t}=0 & \text { in } \widehat{Q} \subset \Omega \times] 0, T[ \\
u(x, t)=0 & \text { in } \widehat{\sum} \\
u(x, 0)=u^{0}(x) & \text { in } \Omega_{0}
\end{array}
$$

satisfying $u \in L^{\infty}\left(0, T ; H_{0}^{1}\left(\Omega_{t}\right)\right), u_{t} \in L^{\infty}\left(0, T ; H_{0}^{1}\left(\Omega_{t}\right)\right)$ and

$$
\int_{\widehat{Q}} u_{t} \phi d x d t-\int_{\widehat{Q}} \sigma(u) \phi_{x} d x d t+\int_{\widehat{Q}} u_{x t} \phi_{x} d x d t=0, \forall \phi \in L^{2}\left(0, T ; H_{0}^{1}\left(\Omega_{t}\right)\right)
$$

$u(x, 0)=u^{0}(x)$. This result is summarized in the following theorem:

Theorem 4.1. For each $u^{0} \in H_{0}^{1}\left(\Omega_{0}\right) \cap H^{2}\left(\Omega_{0}\right)$, and $\widehat{Q}$ a mixed domain satisfying the hypothesis (H5), there exists a function $u: \widehat{Q} \rightarrow \mathbf{R}$, satisfying $u \in$ $L^{\infty}\left(0, T ; H_{0}^{1}\left(\Omega_{t}\right)\right), u_{t} \in L^{\infty}\left(0, T ; H_{0}^{1}\left(\Omega_{t}\right)\right)$ and

$$
\begin{gathered}
\int_{\widehat{Q}} u_{t} \phi d x d t \quad-\int_{\widehat{Q}} \sigma(u) \phi_{x} d x d t+\int_{\widehat{Q}} u_{x t} \phi_{x} d x d t=0, \\
\text { for all } \phi \in L^{2}\left(0, T ; H_{0}^{1}\left(\Omega_{t}\right)\right) \\
u(x, 0)=u^{0}(x), \text { for all } x \in \Omega_{0} .
\end{gathered}
$$

\section{Acknowlegement}

The authors are very grateful to the referee for his observations which improved the value of this article. 


\section{References}

[1] J. Avrin, J.A. Goldstein, Global existence for the Bejamin-Bona-Mahony equation in arbitrary dimensions, Nonlinear Analysis TMA, 9 (1985), 861-865.

[2] R.K. Barreto, C.S.Q. Caldas, P. Gamboa, J. Limaco, On the Rosenau and Benjamin-Bona-Mahony equations in domains with moving boundary, Electronic J. of Diferential Equations, 35 (2004), 12 pp.

[3] T.B. Benjamin, J.L. Bona, J.J. Mahony, Model equations for long waves in nonlinear dispersive sistems, Philos. Trans. Royal Soc. London Ser. A, 272 (1972), 47-78.

[4] J.L. Bona, P.J. Bryant, A mathematical model for long waves generated by wavemakers in non-linear dispersive systems, Proc. Camb. Phil. Soc., 73, (1973), 391-405.

[5] J.L. Bona, V.A. Dougalis, An initial boundary-value problem for a model equation for propagation of long waves, J. Math. Analysis Appl., 75 (1980), 503-522.

[6] C.S.Q. Caldas, J. Limaco, K. Barreto, Linear thermoelastic system in noncylindrical domains, Funckcialaj Ekvacio, 1 (1999), 115-127.

[7] A.T. Cousin, N.A. Larkin, Kuramoto Sivashinski equation in domains with moving boundaries, Port. Math. (N.S.), 59, No. 3 (2002), 336-349.

[8] J.A. Goldstein, Mixed problems for the generalized Benjamin-Bona-Mahony equation, Nonlinear Analysis TMA, 4 (1980), 665-675.

[9] J.L. Lions, Une remarque sur les problèmes $\mathrm{d}^{\prime}$ èvolution linéaires dans des domaines non cylindriques, Revue Roumaine de Math. Pures et Appliquèes, 9 (1964), 11-18.

[10] I.S. Liu, M.A. Rincon, Effect of moving boundaries on the vibrating elastic string, Applied Numerical Mathematics 47, No. 2 (2003), 159-172.

[11] L.A. Medeiros, J. Limaco, Kirchhoff-Carrier elastic strings in non cylindrical domains, Port. Math. (N.S.), 56 (1999), 1-36.

[12] L.A. Medeiros, M. Milla Miranda, Weak solutions for a nonlinear dispersive equations, Journal of Mathematical Analysis and Applications, 59, No. 3 (1977), 432-441.

[13] M.A. Park, On the Rosenau equation, Mat. Aplic. Comp. 9 (1990), 145-152.

[14] P.H. Rosenau, Dynamics of dense discrete systems, Prog. Theoretical Phys., 79 (1988), 1028-1042.

[15] B.S. Santos, J. Limaco, M.A. Rincon, Numerical method, existence and uniqueness for thermoelasticity system with moving boundary. Computational $\& 3 A p$ plied Mathematics, 24, No. 3 (2005), 1-22. 